\title{
Imaging Evidence and Recommendations for Traumatic Brain Injury: Advanced Neuro- and Neurovascular Imaging Techniques
}

M. Wintermark, P.C. Sanelli, Y. Anzai, A.J. Tsiouris, and C.T. Whitlow, on behalf of the American

College of Radiology Head Injury Institute

\begin{abstract}
SUMMARY: Neuroimaging plays a critical role in the evaluation of patients with traumatic brain injury, with NCCT as the first-line of imaging for patients with traumatic brain injury and MR imaging being recommended in specific settings. Advanced neuroimaging techniques, including MR imaging DTI, blood oxygen level-dependent fMRI, MR spectroscopy, perfusion imaging, PET/SPECT, and magnetoencephalography, are of particular interest in identifying further injury in patients with traumatic brain injury when conventional NCCT and MR imaging findings are normal, as well as for prognostication in patients with persistent symptoms. These advanced neuroimaging techniques are currently under investigation in an attempt to optimize them and substantiate their clinical relevance in individual patients. However, the data currently available confine their use to the research arena for group comparisons, and there remains insufficient evidence at the time of this writing to conclude that these advanced techniques can be used for routine clinical use at the individual patient level. TBI imaging is a rapidly evolving field, and a number of the recommendations presented will be updated in the future to reflect the advances in medical knowledge.
\end{abstract}

ABBREVIATIONS: $B O L D=$ blood oxygen level-dependent; $\mathrm{FA}=$ fractional anisotropy; $\mathrm{MD}=$ mean diffusivity; $\mathrm{MEG}=$ magnetoencephalography; $\mathrm{TB}$ = traumatic brain injury

$\mathbf{T}$ raumatic brain injury (TBI) is one of the most common neurologic disorders, currently affecting 1.7 million Americans each year. ${ }^{1,2}$ The incidence of TBI, especially mild TBI, is underestimated ${ }^{3}$ because patients frequently dismiss their symptoms and never present to the emergency department or they believe that the admission of symptoms may compromise their work situation (eg, athletes, military ${ }^{4}$ ). Although most patients (nearly $80 \%$ ) with diagnosed TBI are treated and released from the emergency department, ${ }^{5}$ the remaining $20 \%$ have more severe injuries

From the Division of Neuroradiology (M.W.), Stanford University, Palo Alto, California; Department of Radiology (P.C.S.), North Shore-LIJ Health System, Manhasset, New York; Department of Radiology (Y.A.), University of Washington, Seattle Washington; Department of Radiology (A.J.T.), Weill Cornell Medical College, New York-Presbyterian Hospital, New York, New York; and Department of Radiology and Translational Science Institute (C.T.W.), Wake Forest School of Medicine, Winston-Salem, North Carolina.

M. Wintermark and P.C. Sanelli are co-first authors of this article.

This article is endorsed by the American College of Radiology Head Injury Institute, the American Society of Neuroradiology, the American Society of Functional Neuroradiology, and the American Society of Pediatric Neuroradiology. Another article addressing the evidence and recommendations for conventional neuroimaging techniques in traumatic brain injury is published in Wintermark M, Sanelli PC, Anzai Y, on behalf of the American College of Radiology Head Injury Institute. Imaging evidence and recommendations for traumatic brain injury: conventional neuroimaging techniques. J Am Coll Radiol; http://dx.doi.org/10.1016/j.jacr.2014.10.014

Please address correspondence to Max Wintermark, MD, MAS, Stanford University, Department of Radiology, Neuroradiology Division, 300 Pasteur Dr, Room S047, Stanford, CA 94305-5105; e-mail: Max.Wintermark@gmail.com

- Indicates open access to non-subscribers at www.ajnr.org

http://dx.doi.org/10.3174/ajnr.A4181 resulting in approximately 275,000 hospitalizations and 52,000 deaths each year. Furthermore, TBI contributes to one-third of all injury-related deaths in the United States. The economic cost of TBI was estimated at $\$ 76.3$ billion in 2010 ( $\$ 11.5$ billion in direct medical costs and $\$ 64.8$ billion in indirect costs such as lost wages, lost productivity, and nonmedical expenditures). ${ }^{6}$ Moreover, affected military veterans generate an annual cost of $\$ 11,700$ in medical treatment per patient compared with $\$ 2,400$ in TBI-free veterans. ${ }^{7}$ Leading causes of TBI in the general population include falls, motor vehicle collisions, assaults, and sports-related injuries.

Advanced neuroimaging techniques, including MR imaging DTI, blood oxygen level-dependent (BOLD) fMRI, MR spectroscopy, perfusion imaging, PET/SPECT, and magnetoencephalography (MEG), are of particular interest in identifying further injury in patients with TBI when conventional NCCT and MR imaging findings are normal, as well as for prognostication in patients with persistent symptoms. Based on the National Institute for Health and Care Excellence (http://www.nice.org.uk), adapted from the Oxford Centre for Evidence-Based Medicine (http://www.cebm.net) Levels of Evidence (2001), we indicated the quality of publications for diagnostic tests and interventions by assigning stratified and preferential levels of evidence (Table 1) and classes of recommendations (Table 2). Overall, at the time of writing this article, there is insufficient evidence supporting the routine clinical use of advanced neuroimaging for diagnosis and/or prognostication at the individual patient level (class IIb recommendation). 
Table 1: Levels of evidence for studies of the accuracy of diagnostic tests $^{\mathrm{a}}$

\begin{tabular}{|c|c|}
\hline $\begin{array}{l}\text { Levels of } \\
\text { Evidence }\end{array}$ & Type of Evidence \\
\hline la & Systematic review (with homogeneity) ${ }^{\mathrm{b}}$ of level-1 studies ${ }^{\mathrm{c}}$ \\
\hline $\mathrm{lb}$ & Level-1 studies ${ }^{c}$ \\
\hline \multirow[t]{2}{*}{ II } & Level-2 studies ${ }^{d}$ \\
\hline & Systematic reviews of level-2 studies \\
\hline \multirow[t]{2}{*}{ III } & Level-3 studies ${ }^{\mathrm{e}}$ \\
\hline & Systematic reviews of level-3 studies \\
\hline IV & $\begin{array}{l}\text { Consensus, expert committee reports or opinions and/or } \\
\text { clinical experience without explicit critical appraisal; } \\
\text { or based on physiology, bench research, or "first } \\
\text { principles" }\end{array}$ \\
\hline
\end{tabular}

adapted from The Oxford Centre for Evidence-Based Medicine Levels of Evidence (2001) and the Centre for Reviews and Dissemination Report Number 4 (2001). Copyright National Institute for Health and Care Excellence February 2004.

${ }^{b}$ Homogeneity means there are no or minor variations in the directions and degrees

of results between individual studies that are included in the systematic review.

' Level-1 studies are studies:

- that use a blind comparison of the test with a validated reference standard.

- in a sample of patients that reflects the population to whom the test would apply.

${ }^{d}$ Level-2 studies are studies that have only 1 of the following:

- narrow population (the sample does not reflect the population to whom the test would apply).

- use a poor reference standard (defined as that where the "test" is included in the "reference," or where the "testing" affects the "reference").

- the comparison between the test and reference standard is not blind.

- case-control studies.

${ }^{\mathrm{e}}$ Level-3 studies are studies that have at least 2 or 3 of the features listed above.

Table 2: Classification of recommendation $\mathrm{s}^{\mathrm{a}}$

\begin{tabular}{cc}
\hline Class I & $\begin{array}{c}\text { Conditions for which there is evidence for and/or } \\
\text { general agreement that a procedure or treatment is } \\
\text { beneficial, useful, and effective } \\
\text { Conditions for which there is conflicting evidence } \\
\text { and/or a divergence of opinion about the } \\
\text { usefulness/efficacy of a procedure or treatment } \\
\text { Weight of evidence/opinion is in favor of usefulness/ } \\
\text { efficacy }\end{array}$ \\
Class IIa & $\begin{array}{l}\text { Usefulness/efficacy is less well-established } \\
\text { Conditions for which there is evidence and/or general } \\
\text { agreement that a procedure/treatment is not } \\
\text { Class III } \\
\text { be harmful }\end{array}$ \\
\hline
\end{tabular}

${ }^{a}$ From the American Heart Association Evidence-Based Scoring System.

To date, advanced neuroimaging techniques have been utilized primarily for research, by using a variety of statistical and numeric approaches to assess for differences between study groups. Validated diagnostic tools to interpret advanced neuroimaging techniques within a single patient do not presently exist. The main challenges for developing these tools include the following: 1) lack of large-scale, age-stratified normal data with available advanced neuroimaging techniques by using standardized protocols developed with consensus by clinical and research communities, 2) lack of clear patterns of injury that are predictive of clinical and neuropsychological deficits, and 3) poor definition of standard approaches to account for technical differences between clinical scanners that may introduce artifactual false-positives or -negatives into an assessment.

\section{Advanced Diffusion Imaging Techniques, Including Diffusion Tensor Imaging}

Diffusion tensor imaging ${ }^{8}$ is a well-established model using data derived from directionally encoded MR diffusion-weighted imaging, estimating the overall direction and degree of restriction of water diffusion. In DTI, each voxel has one or more pairs of parameters: a rate of diffusion and a preferred direction of diffusion, described in terms of 3D space, for which that parameter is valid. There is a variety of ways that DTI can quantify the diffusion ellipsoid, the most common of which is to compute scalar metrics, comprising fractional anisotropy (FA) for measuring ellipsoid shape and mean diffusivity (MD) for measuring ellipsoid size. TBI-associated changes in DTI scalar metrics have been well-documented, FA in particular. ${ }^{9-14}$ In addition to measuring a variety of DTI metrics, data processing and analysis techniques have been developed to utilize these data for visualizing abnormalities in gray matter and white matter tracts. ${ }^{15,16}$

With regard to white matter tracts, the diffusion tensor model is not capable of resolving multiple fiber orientations within a voxel, and improved diffusion models and analysis methods are actively being developed. High-angular-resolution diffusion imaging $^{17}$ acquisitions use significantly more diffusion directions compared with many DTI protocols (for instance, 32 or higher). Diffusion kurtosis imaging ${ }^{18}$ uses a minimum of 3 b-values (instead of the typical 2 for DTI, eg, 0, 1000, 3000), providing additional metrics related to non-Gaussian (kurtosis) diffusion that can provide a more physiologic basis for white matter modeling ${ }^{19}$ and for resolving fiber crossings. ${ }^{20}$ Diffusion spectroscopy imaging ${ }^{21}$ requires a very comprehensive sampling scheme, making it sensitive to intravoxel heterogeneities in diffusion directions caused by crossing fiber tracts and thus allows more accurate mapping of axonal trajectories than DTI-based approaches. QBall imaging ${ }^{22}$ attempts a similar granular mapping resolution by using a much smaller diffusion sampling scheme. Newer acquisition methods, such as multiband, ${ }^{23}$ and continued improvement in clinical scanner gradient performance, achieving maximum gradient strengths as high as $80 \mathrm{mT} / \mathrm{m}$, are being developed that allow for faster scan times, making advanced diffusion techniques easier to implement as part of routine clinical protocols.

As mentioned above, DTI cannot resolve crossing fibers, and FA normally decreases in regions of crossing fibers. Methods for resolving crossing fibers include the neurite orientation dispersion and density imaging model ${ }^{24}$ (which derives a measurement of intracellular volume fraction, a potential replacement of fractional anisotropy that should be less confounded by crossing fibers), Q-ball imaging 22 (which assumes a Gaussian model), and constrained spheric deconvolution ${ }^{25}$ (which creates a model based on the actual diffusion acquisition that is used to perform deconvolution and produces very sharp fiber-orientation distribution functions). Further studies, however, are required to determine whether one of these approaches has a clear advantage compared with the others. Improvements in data quality with higher order eddy current correction, ${ }^{26}$ distortion correction, and high-order shimming beyond second order should further improve data fidelity and increase the reliability of subsequent data processing.

Evidence and Recommendations for Advanced Diffusion Imaging Techniques, including Diffusion Tensor Imaging. Most studies of $\mathrm{TBI}$ have reported decreases in FA and increases in $\mathrm{MD}$, thought to be secondary to demyelination or disruption of tissue microstructural integrity. ${ }^{27-36}$ Some studies have reported both trauma-related decreases and increases in FA, particularly in the subacute phase postinjury. New evidence is converging to suggest 
a more complex pattern of trauma-related changes in the white matter. Indeed many studies are reporting that FA values increase in both acute and chronic phases of injury. ${ }^{12,37-41}$ Variability in the data regarding the direction of TBI-related change for common DTI metrics may reflect heterogeneity within cohort studies, including variation in TBI severity, variability in spatial injury location, inconsistent imaging parameters, and marked differences between the time of injury and imaging. ${ }^{42}$ It is important to note that alterations in DTI metrics including FA are not specific to TBI and have also been documented in a wide variety of CNS disorders, particularly those that affect white matter. ${ }^{43}$ When these issues are considered altogether, they suggest a need for more studies to translate this advanced imaging method into a clinically useful and applicable tool for TBI.

Currently, there is evidence from group analyses that DTI can identify TBI-associated changes in the brain across a range of injury severity, from mild to severe TBI. Evidence also suggests that DTI has the sensitivity necessary to detect acute and chronic TBI-associated changes in the brain, some of which correlate with injury outcomes. ${ }^{44}$ These data, however, are based primarily upon group analyses, and there is insufficient evidence at the time of writing this article that DTI can be used for routine clinical diagnosis and/or prognostication at the individual patient level. ${ }^{41,44,45}$ Even though a few studies have reported $z$ score methods for individual patient TBI evaluation, there remains insufficient evidence at the time of writing to suggest that these methods are valid, sensitive, and specific for routine clinical evaluation of TBI at the individual patient level (class IIb recommendation).

\section{Functional MR Imaging}

Functional MR imaging relies on blood oxygen level-dependent imaging and the coupling between $\mathrm{CBF} /$ metabolism and neuronal activity. ${ }^{46-50}$ As metabolic demand increases, there are local increases in CBF, as well as dynamic and related metabolic changes in glucose and oxygen metabolism. ${ }^{46-50}$ Transient local increases in $\mathrm{CBF}$ and metabolism lead to changes in the ratio of oxygenated-to-deoxygenated hemoglobin, which, in turn, affects the MR imaging signal response. ${ }^{46-50}$ BOLD imaging offers millimeter-scale spatial resolution but poor temporal resolution due to the relatively slow hemodynamic response associated with neural activity.

BOLD fMRI methods for investigating TBI have utilized taskbased methods, particularly working memory paradigms. Taskbased methods require participation of the research subject to identify activation of brain regions thought to drive or be associated with task performance. Task-free resting-state BOLD fMRI techniques have also been used, with the advantage of being able to evaluate distributed whole-brain networks without requiring overt behavioral output from subjects. These methods rely upon identification of fluctuations in low-frequency BOLD waveforms throughout the brain, which are thought to reflect underlying neural activity that occurs in the absence of active task performance. Functional connectivity between brain regions is operationally defined as a strong interregional correlation in these lowfrequency BOLD waveforms, which is the basis for identifying functionally connected whole-brain networks comprising multi- ple spatially distributed brain regions. A variety of techniques can be used to study distributed whole-brain networks, such as independent component analysis/seed-based methods. Network properties of the brain can be further characterized by using graph theoretic analysis to identify changes in network function, such as local and global efficiency, as well as small-worldness, among many other network connectivity metrics.

Evidence and Recommendations for Functional MR Imaging. fMRI methods demonstrate great potential for evaluating brain subsystems that may underlie TBI-associated behavioral and cognitive impairment, including the detection of whole-brain changes in functional connectivity across a variety of brain networks, as well as more focused task-specific changes in functional activity among targeted brain subregions. Only a few behavioral/cognitive domains have been evaluated with task-based studies, and only a few functional networks, primarily the default mode, have been evaluated with resting-state techniques, which limits the generalizability of these methods to the breadth of TBI-associated sequelae. ${ }^{41}$ Important limitations have been raised regarding the use of BOLD fMRI techniques for accurately characterizing changes in patients with TBI because of the possibility that brain injury might uncouple CBF and neural activity. ${ }^{51}$ Indeed, there is evidence that TBI is associated with reductions or increases in $\mathrm{CBF}$ that have been described during the acute stages of injury. ${ }^{51,52}$

There is insufficient evidence at the time of this writing that fMRI based on BOLD techniques can be used for routine clinical TBI diagnosis and/or prognostication at the individual patient level (class IIb recommendation).

\section{MR Spectroscopy}

MR spectroscopy is governed by the same physical principles of magnetism as MR imaging. While MR imaging data are analyzed in the time domain to obtain $\mathrm{T} 1$ and $\mathrm{T} 2$ relaxation times and then processed to generate an anatomic image, MR spectroscopy data are converted to frequency domain information and processed to form a spectrum of the signal intensities of different brain metabolites according to their Larmor resonance frequencies.

MR spectroscopy can be performed utilizing single-voxel spectroscopy, or 2D or 3D multivoxel chemical shift techniques (MR spectroscopic imaging or chemical shift imaging). Singlevoxel spectroscopy has a greater signal-to-noise ratio and is more robust, but only a single spectrum is obtained and the volume of interest placement is crucial. Chemical shift imaging limits sampling error by covering a much larger area, at the expense of a lower signal-to-noise ratio and longer scan times. The 2 most widely used MR spectroscopy techniques are point-resolved spectroscopy sequence and the stimulated echo acquisition mode. Commonly quantified brain metabolites with intermediate (TE $=$ $144 \mathrm{~ms}$ ) to long (TE $=280 \mathrm{~ms}$ ) TEs are $\mathrm{N}$-acetylaspartate (NAA) for neuronal integrity, creatine $(\mathrm{Cr})$ for cellular energy/attenuation, choline (Cho) for membrane turnover, and lactate for anaerobic metabolism. At shorter TEs $(\mathrm{TE}=35 \mathrm{~ms})$, metabolites with shorter T2 relaxation times can be detected, such as glutamate/glutamine (Glx), which are excitatory amino acids released after brain injury, ${ }^{53}$ and myo-inositol, thought to be a marker of astroglial proliferation. ${ }^{54}$ Each brain metabolite resonates at a 
specific frequency and is plotted at a known chemical shift (measured in parts per million) on a graphic spectrum. The area under each spectral peak corresponds to the metabolite concentration and can be reported as a ratio to $\mathrm{Cr}$ (such as NAA/Cr and Cho/ $\mathrm{Cr}$ ); $\mathrm{Cr}$ has been used as an internal standard since it was thought to remain relatively constant. It is now known that $\mathrm{Cr}$ may change in certain conditions ${ }^{55-57}$ including $\mathrm{TBI}^{58}$; however, Cr-based ratios remain useful when comparing serial measurements or data between institutions. Methods for absolute metabolite quantitation are now used routinely, by using water as an internal reference or phantoms containing known metabolite concentrations. ${ }^{59-61}$ When imaging children, normal age-matched controls must be used, since brain metabolites change with maturation and myelination, rapidly in the first year of life, and then continue more slowly through adolescence. ${ }^{62-66}$

Evidence and Recommendations for MR Spectroscopy. There is significant heterogeneity in the literature that is, in part, related to the inclusion of patients with variable TBI severity, spatial injury locations, differences between time of injury and imaging, and analysis with various MR spectroscopy techniques. There are currently no large prospective studies examining the sensitivity and specificity of MR spectroscopy in the setting of TBI. A few small studies in patients with moderate-to-severe TBI show promise in the utility of MR spectroscopy as prognostic for outcomes. ${ }^{67,68}$

In mild TBI, the most common finding is a widespread decrease in gray matter and white matter NAA. ${ }^{69-75}$ However, disparity exists between studies in the quantification of Cho in TBI; some studies have found increased Cho in various regions in the brain parenchyma, ${ }^{70,73,76}$ while others have reported the absence of statistical changes in Cho. ${ }^{58,74,75,77} \mathrm{~A}$ few recent small longitudinal prospective controlled cohort studies have shown an initial decrease in NAA, and increase in Cr and Glx in the white matter, but decreased Glx in the gray matter. In all cases, NAA, Cr, and Glx returned to normal levels by the end of the study, suggesting recovery. However, in a recent pediatric study, initial NAA levels did not change following concussion. ${ }^{78}$ A recent controlled crosssectional prospective study that included patients in the early subacute, late subacute, and chronic stages of mild TBI revealed decreasing trends in thalamic NAA/Cr levels at the early subacute stage and decreases in Cho/Cr occurring in the thalamus and centrum semiovale in the late subacute stage. Positive correlations between early subacute $\mathrm{Cr}$ levels in the centrum semiovale and chronic cognitive performance on neuropsychiatric evaluation were noted. Although this provides some insights into brain biochemistry changes at a population level, the authors noted that the applicability of these findings to an individual subject still requires careful examination of data from a larger population. ${ }^{79}$

Studies of MR spectroscopy in chronic mild TBI have found decreases in NAA in the splenium of the corpus callosum, ${ }^{80}$ centrum semiovale, ${ }^{54}$ and frontal white matter. ${ }^{75}$ Chemical shift imaging $^{70,77,81}$ and whole-brain NAA studies ${ }^{69}$ have also demonstrated decreases in NAA in the white matter. Changes found in Cho, a marker for cellular proliferation or tissue damage, may reflect diffuse axonal injury. ${ }^{54,70}$ In the acute phase of head injury, choline-containing metabolites may be released as a result of shear injury and damage to cell membranes and myelin. ${ }^{67}$ In chronic brain injury, the mechanism for increased Cho is more likely diffuse glial proliferation, corroborated by elevated myoinositol that persists for months after injury. ${ }^{82}$ More recent studies have shown changes in the energy marker $\mathrm{Cr}^{58,83}$; if $\mathrm{Cr}$ is affected by mild TBI, metabolite ratio measurements would not be accurate because it would be difficult to assess if changes were due to the metabolite of interest or in Cr itself.

A number of small prospective cross-sectional and case-control longitudinal cohort studies have associated MR spectroscopy metabolite levels with neuropsychological outcomes in the moderate-to-severe TBI population. Most (but not all) of these studies have demonstrated decreased absolute NAA and NAA/Cr ratios and decreased NAA to correlate with poor outcomes. ${ }^{54,67,84-86}$ Decreased NAA/Cr ratios have been detected in the splenium of the corpus callosum and, to a lesser, extent in the lobar white matter. ${ }^{80}$

In pediatric patients with accidental and nonaccidental TBI, MR spectroscopy has shown potential for providing early prognostic information regarding clinical outcomes. ${ }^{62,87-94}$ Reduced NAA has been correlated with impaired long-term neuropsychological function in children. ${ }^{89,90,92,94}$ Elevated total Cho has also been described and may be related to diffuse axonal injury and/or repair. ${ }^{84,95}$ Studies have shown that elevated lactate levels are more common following nonaccidental $\mathrm{TBI}^{62,87}$ and are strongly correlated with poor outcomes. In children, myo-inositol has been shown to increase as a result of glial proliferation and has also been correlated with poor outcomes after TBI. ${ }^{82,96}$

Currently, there remains insufficient evidence at the time of writing to conclude that MR spectroscopy is sufficiently sensitive and specific for routine clinical use at the individual patient level (class IIb recommendation).

\section{Magnetoencephalography}

In MEG, neuromagnetometers surround the head with hundreds of sensors connected to superconducting quantum interference devices that are cooled to $4^{\circ}$ Kelvin with liquid helium. The output of each sensor is a waveform that reveals local magnetic fluctuations and how they change with time. These fluctuations are the result of synchronous postsynaptic intracellular electric currents produced by pyramidal neurons of the cerebral cortex that are accompanied by perpendicularly oriented magnetic fields. ${ }^{97,98}$ Because the net current of pyramidal neuron depolarization is oriented toward the cortical surface, MEG is most sensitive for detecting signals arising from sulcal walls, which are directed perpendicular to the skull. ${ }^{98,99}$ MEG has intrinsically high temporal resolution, limited only by the sampling frequency of the electronics, ${ }^{100}$ which is an advantage over other functional neuroimaging methods, such as fMRI. However, MEG has inferior spatial resolution, largely because a single cortical source of signal can be detected by multiple adjacent scalp sensors. As such, data must be processed to translate sensor signal back to the source space.

Evidence and Recommendations for Magnetoencephalography. There is insufficient evidence at the time of writing that MEG can be used for routine clinical TBI diagnosis and/or prognostication at the individual patient level (class IIb recommendation). Indeed, only a few studies have used MEG to evaluate the effects of TBI ${ }^{101-104}$; therefore, more work is necessary to define the utility and capabilities of this imaging technique. Very few 
medical centers have modern neuromagnetometers, and analysis of MEG data requires advanced expertise in signal processing, which limits its widespread implementation.

\section{Perfusion Imaging}

Brain perfusion in patients with TBI has been imaged by using different techniques, including stable xenon-enhanced CT, ${ }^{105,106}$ single-photon emission CT (SPECT), ${ }^{107-113}$ PET, ${ }^{114,115}$ perfusion $\mathrm{CT}^{116-120}$ and perfusion-weighted MR imaging, including dynamic susceptibility contrast, ${ }^{121,122}$ and arterial spin-labeling. ${ }^{123,124}$ These brain perfusion imaging techniques each have different drawbacks. SPECT and dynamic susceptibility contrast only generate qualitative comparisons between the right and left hemispheres. ${ }^{125}$ Stable xenon-enhanced CT, perfusion CT, and arterial spin-labeling are quantitatively accurate. ${ }^{126,127}$

Stable xenon-enhanced CT requires specialized and expensive equipment. A typical study is relatively long, approximately 10 minutes. Side effects, such as respiratory rate decrease, headaches, nausea and vomiting, as well as convulsions, are observed in $4.4 \%$ of patients. Consequently, stable xenon-enhanced CT is difficult to perform, especially in the emergency setting. ${ }^{128}$

Perfusion-weighted MR imaging is also difficult to obtain in the acute setting due to scanner availability and patient contraindications but is an attractive imaging technique for the subacute and chronic phases, especially because it can be combined with other MR imaging sequences sensitive to TBI lesions.

On the other hand, perfusion CT is more readily available in the emergency/acute setting. ${ }^{120}$ It can be implemented in all hospital institutions equipped with CT units and injectors, which are usually available 24/7. It necessitates neither specialized technologists nor extra material, but only requires dedicated postprocessing software. It affords real-time postprocessing, with a complete set of parametric maps typically generated within 5 minutes of completing data acquisition. ${ }^{129,130}$ Perfusion CT can easily be performed as a complement to conventional noncontrast CT (NCCT) of the head and CT angiography (CTA) of the cerebral vasculature and does not interfere with the contrast-enhanced thoracoabdominal CT survey performed in patients with severe TBI. ${ }^{116}$ Patients with severe TBI typically undergo contrast-enhanced chest, abdomen, and pelvis CT routinely to evaluate for aortic injuries. The contrast material administration is performed even in obtunded patients unable to report about possible previous contrast reactions or without knowing about the renal function because the risk associated with these conditions is outweighed significantly by the risk of a missed traumatic aortic injury. The dose of contrast material added for the perfusion CT is minor compared with the dose used for the chest, abdomen, and pelvis.

In patients with severe TBI, perfusion imaging affords insight into regional brain perfusion alterations due to TBI, with the major advantage being detection of regional heterogeneity. ${ }^{116}$ Perfusion imaging has a higher sensitivity for the diagnosis of cerebral contusions at baseline when compared with admission NCCT, with a sensitivity reaching $87.5 \%$ versus $39.6 \%$ for conventional NCCT. ${ }^{16,120,121}$ Perfusion imaging can show changes related to the mass effect caused by an epidural or subdural hematoma and the resolution of these changes after hematoma evacuation. ${ }^{131}$
Perfusion imaging can also identify specific patterns, linked to cerebral edema and intracranial hypertension. ${ }^{117,118}$ Finally, the number of arterial territories with "low" regional cerebral blood volume (CBV) values on perfusion imaging is an independent predictor of the functional outcome and is seen as early as on admission. ${ }^{116}$ The potential repercussions of perfusion imaging on the clinical management of patients with severe TBI remain to be evaluated. However, patients with altered brain perfusion might be considered for more aggressive and early treatment to prevent intracranial hypertension, whereas patients with preserved brain perfusion might benefit from less invasive treatment.

In patients with mild TBI and normal conventional brain imaging findings, perfusion imaging studies have shown scattered perfusion deficits, which are significantly correlated with neuropsychological and neurocognitive impairment, as well as posttraumatic amnesia. ${ }^{109,110,112,119,122,132,133}$

Evidence and Recommendations for Perfusion Imaging. All the studies above have involved only limited numbers of patients, and further research is required to validate the findings described above and determine how relevant they are in the management of individual patients with TBI (class IIb recommendation).

\section{PET and SPECT}

Most studies utilizing PET and SPECT to investigate TBI have focused on cerebral blood flow and metabolism and demonstrated TBI-associated decreases in cohort studies. ${ }^{134-138}$ Recent literature reviews summarizing the possible utility of $\mathrm{PET}^{139}$ and SPECT $^{140}$ in TBI, particularly mild TBI, have drawn this cohort data largely from cross-sectional designs with a smaller number of longitudinal studies also reviewed. Very few randomized controlled trials were discovered when examining the literature. Also, various levels of analytic rigor have been used to identify TBIassociated changes with these imaging methods, including quantitative and visual-based qualitative measures, which are known to have interrater variability. Additionally, very few PET and SPECT studies to date have gone beyond CBF and metabolic mapping to incorporate specific ligands for exploring TBI-related changes at the receptor level, which may open the door to a new generation of sensitive and specific molecular biomarkers of brain injury. ${ }^{141}$ Recent PET amyloid imaging, however, suggests that amyloid levels rise in persons with TBI. ${ }^{142}$ A small study with a combined amyloid tau $(\tau)$ tracer also showed higher levels in those with TBI. ${ }^{143}$ The condition, sometimes referred to as "chronic traumatic encephalopathy," is believed to be progressive tauopathy, seen most commonly in persons with repetitive concussive brain injuries, such as professional athletes and military personnel. ${ }^{144}$ Beta $(\beta)$ amyloid deposition, while seen in $40 \%$ $45 \%$ of chronic traumatic encephalopathy, is not as common as $\tau$ accumulation. ${ }^{145}$ As TBI is a recognized risk factor for cognitive decline and dementia, ${ }^{146}$ advanced neuroimaging will likely continue to provide key insights into this disease process and possible avenues for routine and personalized clinical use. At present, more work must be conducted to translate these powerful neuroimaging methods into useful, routine clinical tools at the individual subject level, for which there is insufficient evidence at the time of writing this article (class IIb recommendation). 


\section{Summary}

1) Advanced neuroimaging techniques, including $M R$ imaging DTI, BOLD fMRI, MR spectroscopy, perfusion imaging, PET/SPECT, and MEG, are of particular interest for patients with mild TBI when conventional NCCT and MR imaging findings are negative. These advanced neuroimaging techniques have shown promising results in group comparison analyses.

2) At the time of writing, there is insufficient evidence supporting the routine clinical use of advanced neuroimaging for diagnosis and/or prognostication at the individual patient level (class IIb recommendation).

\section{Imaging of Neurovascular Trauma}

Traumatic neurovascular injuries are a rare but known occurrence associated with certain types of blunt trauma to the face and head. They occur in approximately $0.1 \%-2 \%$ of patients with severe trauma, with carotid and vertebral arteries being equally affected. ${ }^{147,148}$

The natural history of these lesions is still being elucidated and optimal treatment plans formulated. A grading scheme, called the Denver grading scale, has been proposed by Biffl et $\mathrm{al}^{149}$ :

Grade I: intimal irregularity with $<25 \%$ narrowing

Grade II: dissection with intramural hematoma and $>25 \%$ luminal narrowing

Grade III: pseudoaneurysm

Grade IV: occlusion

Grade V: transection with extravasation, as well as arteriovenous fistula.

Undetected, traumatic neurovascular injuries can cause ischemic stroke and are associated with high mortality rates. Implementation of the screening protocol can virtually eliminate injury-related strokes in patients without primary thromboembolic neurologic deficits. ${ }^{150}$

Several other injuries and injury patterns can be used to identify patients with a high likelihood of concurrent traumatic neurovascular injuries, and these patterns can be used as indications to screen for traumatic neurovascular injuries. Patients with blunt trauma with a high-velocity mechanism, low Glasgow Coma Scale score, high injury severity score, mandible fracture, complex skull fractures, basilar skull fractures (including carotid canal fractures), scalp degloving, any type of cervical spine injury, traumatic brain injury with thoracic injuries, and/or thoracic vascular imaging are at increased risk for blunt neurovascular injuries (evidence level II). ${ }^{148,151-154}$ In patients identified as having these types of concurrent injuries, the overall rate of blunt neurovascular injuries increases up to $27 \%-30 \% .^{147}$

Although digital subtraction angiography is the diagnostic reference standard for detecting blunt neurovascular injuries, a number of retrospective studies and meta-analyses indicate that multidetector CT angiography is an accurate, rapid, noninvasive diagnostic alternative. CTA of the neck extended to include the circle of Willis has a sensitivity and specificity of $90 \%-100 \%$ for diagnosing vascular trauma in blunt vascular injury within the neck (evidence level II). ${ }^{155-159}$ Based on the evidence available, CTA of the neck extended to include the circle of Willis should be the first-line investigation for all patients with suspected vascular trauma and who have no indication for immediate operative intervention (class IIa recommendation). ${ }^{160}$ CTA can be used to grade (in the acute setting) and follow-up (in the subacute and chronic settings) blunt neurovascular injuries, providing significant prognostic information and influencing management decisions. $^{161}$

The application of duplex ultrasonography, MR imaging/angiography, and transesophageal echocardiography has been described, but these imaging modalities are usually more difficult to obtain in the acute setting in patients involved in severe trauma (evidence level III) due to availability of techniques and patient contraindications. For the same reason, 4-vessel DSA is only used in the acute setting in patients with inconclusive CTA interpretation or when an endovascular intervention is considered. ${ }^{150,160}$

The description above pertained to blunt neurovascular injuries but also applies to penetrating neck injury. A comprehensive physical examination, combined with CTA of the neck extended to include the circle of Willis, is adequate for triage to effectively identify or exclude vascular and aerodigestive injury after penetrating neck trauma (evidence level III), with endoscopy and angiography serving as second-line evaluation modalities (class IIa recommendation). ${ }^{162}$ Traumatic neurovascular injuries are treated medically and, when needed, by endovascular approaches (coil embolization, stent placement), with a low rate of immediate and delayed neurovascular complications (evidence level II). ${ }^{163,164}$

\section{Summary}

1) Traumatic neurovascular injuries should be suspected in patients with a high-velocity mechanism, low Glasgow Coma Scale score, high injury severity score, AND mandible fracture, complex skull fractures, basilar skull fractures (including carotid canal fractures), scalp degloving, any type of cervical spine injury, and/or TBI with thoracic injuries, and/or thoracic vascular imaging, as well as in patients with penetrating neck injury (class I recommendation).

2) CTA of the neck extended to include the circle of Willis should be the first-line investigation for all patients with suspected vascular trauma and who have no indication for immediate operative intervention (class IIa recommendation).

3) A relatively high proportion of traumatic neurovascular injuries are treated by endovascular approaches (coil embolization, stent placement), with a low rate of immediate and delayed neurovascular complications.

\section{CONCLUSIONS}

A number of advanced neuroimaging techniques are currently under investigation in an attempt to optimize them and substantiate their clinical relevance in individual patients; however, the data currently available confine their use to the research arena for group comparisons. TBI imaging is a rapidly evolving field, and a number of the recommendations presented will be updated in the future to reflect the advances in medical knowledge. 


\section{APPENDIX}

\section{American College of Radiology Head Injury Institute}

Significant contributors: T. Jason Druzgal, MD, PhD, Department of Radiology, University of Virginia, Charlottesville, Virginia; Alisa D. Gean, MD, Department of Radiology, University of California, and Brain and Spinal Injury Center, San Francisco General Hospital, San Francisco, California; Yvonne W. Lui, MD, Department of Radiology, New York University School of Medicine, New York, New York; Alexander M. Norbash, MD, Department of Radiology, Boston University School of Medicine, Boston, Massachusetts; Cyrus Raji, MD, Department of Radiology, University of California, Los Angeles, Los Angeles, California; David W. Wright, MD, FACEP, Department of Emergency Medicine, Emory University School of Medicine, Atlanta, Georgia; Michael Zeineh, MD, Department of Radiology, Stanford University, Palo Alto, California.

This article was reviewed and approved by: Joseph M. Aulino, MD, Department of Radiology, Vanderbilt University, Nashville, Tennessee; Jeffrey T. Barth, PhD, Department of Psychiatry and Neurobehavioral Sciences, University of Virginia, Charlottesville, Virginia; Michael Brant-Zawadzki, MD, FACR, Hoag Hospital, Newport Beach, California; Asim F. Choudhri, MD, Department of Radiology, University of Tennessee Health Science Center, Memphis, Tennessee; Amanda Corey, MD, Department of Radiology, Emory University School of Medicine, Atlanta, Georgia; Rebecca S. Cornelius, MD, FACR, Departments of Radiology and Otolaryngology, University of Cincinnati Medical Center, Cincinnati, Ohio; Cameron Craddock, PhD, Center for the Developing Brain, Child Mind Institute, New York, New York; Burton P. Drayer, MD, Department of Radiology, Mount Sinai Hospital, New York, New York; Steven Flanagan, MD, Department of Rehabilitation Medicine, New York University Langone Medical Center, New York, New York; Magali Haas, MD, PhD, Orion Bionetworks; Joseph A. Helpern, PhD, Department of Radiology, Medical University of South Carolina, Charleston, South Carolina; Ramona Hicks, PhD, Program Director, Repair and Plasticity, National Institutes of Health/National Institute of Neurological Disorders and Stroke, Bethesda, Maryland; Randy Horton, American College of Radiology Head Injury Institute, Reston, Virginia; Patricia Hudgins, MD, Department of Radiology, Emory University School of Medicine, Atlanta, Georgia; Carolyn Cidis Meltzer, MD, FACR, Department of Radiology and Imaging Sciences, Emory University School of Medicine, Atlanta, Georgia; David Mikulis, MD, PhD, Department of Radiology, University of Toronto, Toronto, Ontario, Canada; Sumit N. Niogi, MD, $\mathrm{PhD}$, Department of Radiology, New York-Presbyterian Hospital, Weill Cornell Medical Center, New York, New York; Michael Rothman, MD, Bethlehem, Pennsylvania; David Seidenwurm, MD, Department of Radiology, Sutter Health, Sacramento, California; Martha Shenton, PhD, Department of Psychiatry, Brigham and Women's Hospital, Harvard Medical School, Boston, Massachusetts; James R. Stone, MD, PhD, Department of Radiology, University of Virginia, Charlottesville, Virginia; Gordon Sze, MD, Department of Radiology, Yale University, New Haven, Connecticut; Elizabeth A. Wilde, PhD, Departments of Physical Medicine and Rehabilitation, Neurology, and Radiology, Baylor College of Medicine, Houston, Texas;
David W. Wright, MD, FACEP, Department of Emergency Medicine, Emory University School of Medicine, Atlanta, Georgia; Robert D. Zimmerman, MD, Department of Radiology, Weill Cornell Medical College, New York-Presbyterian Hospital, New York, New York.

\section{ACKNOWLEDGMENTS}

We would like to thank Laura Coombs and Barbara Seedah from the American College of Radiology for the support they provided in the preparation of this article.

Disclosures: Max Wintermark-UNRELATED: Grants/Grants Pending: GE Healthcare, ${ }^{*}$ Philips Healthcare.* Apostolos J. Tsiouris-UNRELATED: Consultancy: BioClinica, Parexel, Comments: BioClinica and Parexel are contract research organizations for which I perform blinded expert imaging interpretations and reports for oncology pharmaceutical trials; Expert Testimony: I perform expert imaging reviews for medicolegal cases (approximately 5-10 per year). *Money paid to the institution.

\section{REFERENCES}

1. Faul M, Xu L, Wald M, et al. Traumatic Brain Injury in the United States: Emergency Department Visits, Hospitalizations and Deaths 2002-2006. Atlanta: Centers for Disease Control and Prevention, National Center for Injury Prevention and Control; 2010

2. Marin JR, Weaver MD, Yealy DM, et al. Trends in visits for traumatic brain injury to emergency departments in the United States. JAMA 2014;311:1917-19

3. Ilie G, Boak A, Adlaf EM, et al. Prevalence and correlates of traumatic brain injuries among adolescents. JAMA 2013;309:2550-52

4. Gean AD. Brain Injury: Applications from War and Terrorism. Lippincott Williams \& Wilkins; 2014

5. National Center for Injury Prevention and Control. Report to Congress on Mild Traumatic Brain Injury in the United States: Steps to Prevent a Serious Public Health Problem. Atlanta: Centers for Disease Control and Prevention; 2003

6. Centers for Disease Control and Prevention. Injury Prevention and Control: Traumatic Brain Injury. http://www.cdc.gov/traumatic braininjury/. Updated March 6, 2014. Accessed October 30, 2014

7. Congressional Budget Office. The Veterans Health Administration's Treatment of PTSD and Traumatic Brain Injury Among Recent Combat Veterans. http://www.cbo.gov/sites/default/files/cbofiles/ attachments/02-09-PTSD.pdf. February 2012. Accessed October 20, 2014

8. Basser PJ, Mattiello J, LeBihan D. Estimation of the effective selfdiffusion tensor from the NMR spin echo. J Magn Reson B 1994;103:247-54

9. Davenport ND, Lim KO, Armstrong MT, et al. Diffuse and spatially variable white matter disruptions are associated with blast-related mild traumatic brain injury. Neuroimage 2012;59:2017-24

10. Mayer AR, Ling JM, Yang Z, et al. Diffusion abnormalities in pediatric mild traumatic brain injury. J Neurosci 2012;32:17961-69

11. Ling JM, Peña A, Yeo RA, et al. Biomarkers of increased diffusion anisotropy in semi-acute mild traumatic brain injury: a longitudinal perspective. Brain 2012;135:1281-92

12. Wilde EA, McCauley SR, Hunter JV, et al. Diffusion tensor imaging of acute mild traumatic brain injury in adolescents. Neurology 2008;70:948-55

13. Chu Z, Wilde EA, Hunter JV, et al. Voxel-based analysis of diffusion tensor imaging in mild traumatic brain injury in adolescents. AJNR Am J Neuroradiol 2010;31:340-46

14. Mayer AR, Ling J, Mannell MV, et al. A prospective diffusion tensor imaging study in mild traumatic brain injury. Neurology 2010;74:643-50

15. Smith SM, Jenkinson M, Johansen-Berg H, et al. Tract-based spatial statistics: voxelwise analysis of multi-subject diffusion data. $\mathrm{Neu}$ roimage 2006;31:1487-505

16. Bouix $S$, Pasternak $O$, Rathi $Y$, et al. Increased gray matter diffusion 
anisotropy in patients with persistent post-concussive symptoms following mild traumatic brain injury. PLoS One 2013;8:e66205

17. Tuch DS, Reese TG, Wiegell MR, et al. High angular resolution diffusion imaging reveals intravoxel white matter fiber heterogeneity. Magn Reson Med 2002;48:577-82

18. Jensen JH, Helpern JA. MRI quantification of non-Gaussian water diffusion by kurtosis analysis. NMR Biomed 2010;23:698-710

19. Fieremans E, Benitez A, Jensen JH, et al. Novel white matter tract integrity metrics sensitive to Alzheimer disease progression. AJNR Am J Neuroradiol 2013;34:2105-12

20. Jensen JH, Helpern JA, Tabesh A. Leading non-Gaussian corrections for diffusion orientation distribution function. NMR Biomed 2014;27:202-11

21. Wedeen VJ, Hagmann P, Tseng WY, et al. Mapping complex tissue architecture with diffusion spectrum magnetic resonance imaging. Magn Reson Med 2005;54:1377-86

22. Tuch DS. Q-ball imaging. Magnet Reson Med 2004;52:1358-72

23. Feinberg DA, Moeller S, Smith SM, et al. Multiplexed echo planar imaging for sub-second whole brain FMRI and fast diffusion imaging. PLoS One 2010;5:e15710

24. Zhang H, Schneider T, Wheeler-Kingshott CA, et al. NODDI: practical in vivo neurite orientation dispersion and density imaging of the human brain. Neuroimage 2012;61:1000-16

25. Tournier J, Calamante F, Connelly A. Robust determination of the fibre orientation distribution in diffusion MRI: non-negativity constrained super-resolved spherical deconvolution. Neuroimage 2007;35:1459-72

26. Xu D, Maier JK, King KF, et al. Prospective and retrospective high order eddy current mitigation for diffusion weighted echo planar imaging. Magnet Reson Med 2013;70:1293-305

27. Wilde EA, Ramos MA, Yallampalli R, et al. Diffusion tensor imaging of the cingulum bundle in children after traumatic brain injury. Dev Neuropsychol 2010;35:333-51

28. Arfanakis K, Haughton VM, Carew JD, et al. Diffusion tensor MR imaging in diffuse axonal injury. AJNR Am J Neuroradiol 2002;23:794-802

29. Kumar R, Gupta RK, Husain M, et al. Comparative evaluation of corpus callosum DTI metrics in acute mild and moderate traumatic brain injury: its correlation with neuropsychometric tests. Brain Inj 2009;23:675-85

30. Miles L, Grossman RI, Johnson G, et al. Short-term DTI predictors of cognitive dysfunction in mild traumatic brain injury. Brain Inj 2008;22:115-22

31. Newcombe VF, Williams GB, Nortje J, et al. Concordant biology underlies discordant imaging findings: diffusivity behaves differently in grey and white matter post acute neurotrauma. Acta Neurochir Suppl 2008;102:247-51

32. Newcombe VF, Williams GB, Nortje J, et al. Analysis of acute traumatic axonal injury using diffusion tensor imaging. $\mathrm{Br}$ J Neurosurg 2007;21:340-48

33. Wozniak JR, Lim KO. Advances in white matter imaging: a review of in vivo magnetic resonance methodologies and their applicability to the study of development and aging. Neurosci Biobehav Rev 2006;30:762-74

34. Wozniak JR, Krach L, Ward E, et al. Neurocognitive and neuroimaging correlates of pediatric traumatic brain injury: a diffusion tensor imaging (DTI) study. Arch Clin Neuropsychol 2007;22:555-68

35. Aoki Y, Inokuchi R, Gunshin M, et al. Diffusion tensor imaging studies of mild traumatic brain injury: a meta-analysis. J Neurol Neurosurg Psychiatry 2012;83:870-76

36. Brandstack N, Kurki T, Tenovuo O. Quantitative diffusion-tensor tractography of long association tracts in patients with traumatic brain injury without associated findings at routine MR imaging. Radiology 2013;267:231-39

37. McAllister TW, Ford JC, Ji S, et al. Maximum principal strain and strain rate associated with concussion diagnosis correlates with changes in corpus callosum white matter indices. Ann Biomed Eng 2012;40:127-40

38. Henry LC, Tremblay J, Tremblay S, et al. Acute and chronic changes in diffusivity measures after sports concussion. J Neurotrauma 2011;28:2049-59

39. Bazarian JJ, Zhong J, Blyth B, et al. Diffusion tensor imaging detects clinically important axonal damage after mild traumatic brain injury: a pilot study. J Neurotrauma 2007;24:1447-59

40. Bazarian JJ, Zhu T, Blyth B, et al. Subject-specific changes in brain white matter on diffusion tensor imaging after sports-related concussion. Magn Reson Imaging 2012;30:171-80

41. Eierud C, Craddock RC, Fletcher S, et al. Neuroimaging after mild traumatic brain injury: review and meta-analysis. Neuroimage Clin 2014;4:283-94

42. Hulkower M, Poliak D, Rosenbaum S, et al. A decade of DTI in traumatic brain injury: 10 years and $\mathbf{1 0 0}$ articles later. AJNR Am J Neuroradiol 2013;34:2064-74

43. Fakhran S, Yaeger K, Alhilali L. Symptomatic white matter changes in mild traumatic brain injury resemble pathologic features of early Alzheimer dementia. Radiology 2013;269:249-57

44. Shenton M, Hamoda H, Schneiderman J, et al. A review of magnetic resonance imaging and diffusion tensor imaging findings in mild traumatic brain injury. Brain Imaging Behav 2012;6:137-92

45. Niogi SN, Mukherjee P. Diffusion tensor imaging of mild traumatic brain injury. J Head Trauma Rehabil 2010;25:241-55

46. Logothetis NK, Pauls J, Augath M, et al. Neurophysiological investigation of the basis of the fMRI signal. Nature 2001;412:150-57

47. Heeger DJ, Ress D. What does fMRI tell us about neuronal activity? Nat Rev Neurosci 2002;3:142-51

48. Arthurs OJ, Boniface S. How well do we understand the neural origins of the fMRI BOLD signal? Trends Neurosci 2002;25:27-31

49. Attwell $\mathrm{D}$, Iadecola $\mathrm{C}$. The neural basis of functional brain imaging signals. Trends Neurosci 2002;25:621-25

50. Nair DG. About being BOLD. Brain Res Brain Res Rev 2005;50:229-43

51. Jantzen KJ. Functional magnetic resonance imaging of mild traumatic brain injury. J Head Trauma Rehab 2010;25:256-66

52. Freeman JR, Barth JT, Broshek DK, et al. Sports injuries. In: Silver JM, McAllister TW, Yudofsky SC, eds. Textbook of Traumatic Brain Injury. Washington, DC: American Psychiatric Publishing Inc; 2005:453-76

53. Bullock R, Zauner A, Woodward JJ, et al. Factors affecting excitatory amino acid release following severe human head injury. J Neurosurg 1998;89:507-18

54. Garnett MR, Blamire AM, Rajagopalan B, et al. Evidence for cellular damage in normal-appearing white matter correlates with injury severity in patients following traumatic brain injury: a magnetic resonance spectroscopy study. Brain 2000;123(pt 7):1403-09

55. Hattingen E, Raab P, Franz K, et al. Prognostic value of choline and creatine in WHO grade II gliomas. Neuroradiology 2008;50:759-67

56. Inglese M, Li BS, Rusinek H, et al. Diffusely elevated cerebral choline and creatine in relapsing-remitting multiple sclerosis. Magn Reson Med 2003;50:190-95

57. Muñoz Maniega S, Cvoro V, Armitage PA, et al. Choline and creatine are not reliable denominators for calculating metabolite ratios in acute ischemic stroke. Stroke 2008;39:2467-69

58. Gasparovic C, Yeo R, Mannell M, et al. Neurometabolite concentrations in gray and white matter in mild traumatic brain injury: an $1 \mathrm{H}$-magnetic resonance spectroscopy study. J Neurotrauma 2009;26:1635-43

59. Danielsen ER, Michaelis T, Ross BD. Three methods of calibration in quantitative proton MR spectroscopy. J Magn Reson B 1995; 106:287-91

60. Jansen JF, Backes WH, Nicolay K, et al. 1H MR spectroscopy of the brain: absolute quantification of metabolites. Radiology 2006;240:318-32

61. Provencher SW. Estimation of metabolite concentrations from lo- 
calized in vivo proton NMR spectra. Magn Reson Med 1993;30:672-79

62. Holshouser BA, Ashwal S, Luh GY, et al. Proton MR spectroscopy after acute central nervous system injury: outcome prediction in neonates, infants, and children. Radiology 1997;202:487-96

63. Horská A, Kaufmann WE, Brant LJ, et al. In vivo quantitative proton MRSI study of brain development from childhood to adolescence. J Magn Reson Imaging 2002;15:137-43

64. Kreis R, Ernst T, Ross BD. Development of the human brain: invivo quantification of metabolite and water-content with proton magnetic-resonance spectroscopy. Magnet Reson Med 1993;30: 424-37

65. Kreis R, Hofmann L, Kuhlmann B, et al. Brain metabolite composition during early human brain development as measured by quantitative in vivo H-1 magnetic resonance spectroscopy. Magnet Reson Med 2002;48:949-58

66. Pouwels PJW, Brockmann K, Kruse B, et al. Regional age dependence of human brain metabolites from infancy to adulthood as detected by quantitative localized proton MRS. Pediatr Res 1999;46:474-85

67. Ross BD, Ernst T, Kreis R, et al. 1H MRS in acute traumatic brain injury. J Magn Reson Imaging 1998;8:829-40

68. Shutter L, Tong KA, Holshouser BA. Proton MRS in acute traumatic brain injury: role for glutamate/glutamine and choline for outcome prediction. J Neurotrauma 2004;21:1693-705

69. Cohen BA, Inglese M, Rusinek $\mathrm{H}$, et al. Proton MR spectroscopy and MRI-volumetry in mild traumatic brain injury. AJNR Am J Neuroradiol 2007;28:907-13

70. Govindaraju V, Gauger GE, Manley GT, et al. Volumetric proton spectroscopic imaging of mild traumatic brain injury. AJNR Am J Neuroradiol 2004;25:730-37

71. Henry LC, Tremblay S, Boulanger Y, et al. Neurometabolic changes in the acute phase after sports concussions correlate with symptom severity. J Neurotrauma 2010;27:65-76

72. Henry LC, Tremblay S, Leclerc S, et al. Metabolic changes in concussed American football players during the acute and chronic post-injury phases. BMC Neurol 2011;11:105

73. Sarmento E, Moreira P, Brito C, et al. Proton spectroscopy in patients with post-traumatic headache attributed to mild head injury. Headache 2009;49:1345-52

74. Vagnozzi R, Signoretti S, Cristofori L, et al. Assessment of metabolic brain damage and recovery following mild traumatic brain injury: a multicentre, proton magnetic resonance spectroscopic study in concussed patients. Brain 2010;133:3232-42

75. Vagnozzi R, Signoretti S, Tavazzi B, et al. Temporal window of metabolic brain vulnerability to concussion: a pilot $1 \mathrm{H}$-magnetic resonance spectroscopic study in concussed athletes-part III. Neurosurgery 2008;62:1286-95; discussion 1295-96

76. Govind V, Gold S, Kaliannan K, et al. Whole-brain proton MR spectroscopic imaging of mild-to-moderate traumatic brain injury and correlation with neuropsychological deficits. $J \mathrm{Neu}$ rotrauma 2010;27:483-96

77. Kirov I, Fleysher L, Babb JS, et al. Characterizing 'mild' in traumatic brain injury with proton MR spectroscopy in the thalamus: initial findings. Brain Inj 2007;21:1147-54

78. Maugans TA, Farley C, Altaye M, et al. Pediatric sports-related concussion produces cerebral blood flow alterations. Pediatrics 2012;129:28-37

79. George EO, Roys S, Sours C, et al. Longitudinal and prognostic evaluation of mild traumatic brain injury: a $1 \mathrm{H}$-magnetic resonance spectroscopy study. J Neurotrauma 2014;31:1018-28

80. Cecil KM, Hills EC, Sandel ME, et al. Proton magnetic resonance spectroscopy for detection of axonal injury in the splenium of the corpus callosum of brain-injured patients. J Neurosurg 1998;88:795-801

81. Cimatti M. Assessment of metabolic cerebral damage using proton magnetic resonance spectroscopy in mild traumatic brain injury. J Neurosurg Sci 2006;50:83-88
82. Ashwal S, Holshouser B, Tong K, et al. Proton spectroscopy detected myoinositol in children with traumatic brain injury. Pediatr Res 2004;56:630-38

83. Yeo RA, Gasparovic C, Merideth F, et al. A longitudinal proton magnetic resonance spectroscopy study of mild traumatic brain injury. J Neurotrauma 2011;28:1-11

84. Brooks WM, Stidley CA, Petropoulos H, et al. Metabolic and cognitive response to human traumatic brain injury: a quantitative proton magnetic resonance study. J Neurotrauma 2000;17:629-40

85. Friedman SD, Brooks WM, Jung RE, et al. Quantitative proton MRS predicts outcome after traumatic brain injury. Neurology 1999;52:1384-91

86. Friedman SD, Brooks WM, Jung RE, et al. Proton MR spectroscopic findings correspond to neuropsychological function in traumatic brain injury. AJNR Am J Neuroradiol 1998;19:1879-85

87. Aaen GS, Holshouser BA, Sheridan C, et al. Magnetic resonance spectroscopy predicts outcomes for children with nonaccidental trauma. Pediatrics 2010;125:295-303

88. Ashwal S, Holshouser BA, Shu SK, et al. Predictive value of proton magnetic resonance spectroscopy in pediatric closed head injury. Pediatr Neurol 2000;23:114-25

89. Babikian T, Freier MC, Ashwal S, et al. MR spectroscopy: predicting long-term neuropsychological outcome following pediatric TBI. $J$ Magn Reson Imaging 2006;24:801-11

90. Brenner T, Freier MC, Holshouser BA, et al. Predicting neuropsychologic outcome after traumatic brain injury in children. Pediatr Neurol 2003;28:104-14

91. Holshouser BA, Tong KA, Ashwal S. Proton MR spectroscopic imaging depicts diffuse axonal injury in children with traumatic brain injury. AJNR Am J Neuroradiol 2005;26:1276-85

92. Hunter JV, Thornton RJ, Wang ZJ, et al. Late proton MR spectroscopy in children after traumatic brain injury: correlation with cognitive outcomes. AJNR Am J Neuroradiol 2005;26:482-88

93. Makoroff KL, Cecil KM, Care M, et al. Elevated lactate as an early marker of brain injury in inflicted traumatic brain injury. Pediatr Radiol 2005;35:668-76

94. Yeo RA, Phillips JP, Jung RE, et al. Magnetic resonance spectroscopy detects brain injury and predicts cognitive functioning in children with brain injuries. J Neurotrauma 2006;23:1427-35

95. Holshouser BA, Tong KA, Ashwal S, et al. Prospective longitudinal proton magnetic resonance spectroscopic imaging in adult traumatic brain injury. J Magn Reson Imaging 2006;24:33-40

96. Garnett MR, Corkill RG, Blamire AM, et al. Altered cellular metabolism following traumatic brain injury: a magnetic resonance spectroscopy study. J Neurotrauma 2001;18:231-40

97. Hari R, Forss N. Magnetoencephalography in the study of human somatosensory cortical processing. Philos Trans R Soc Lond B Biol Sci 1999;354:1145-54

98. Hari R, Salmelin R. Magnetoencephalography: from SQUIDs to neuroscience-Neuroimage 20th anniversary special edition. Neuroimage 2012;61:386-96

99. Hamalainen M, Hari R, Ilmoniemi RJ, et al. Magnetoencephalography - theory, instrumentation, and applications to noninvasive studies of the working human brain. Rev Mod Phys 1993;65:413-97

100. Stam CJ. Use of magnetoencephalography (MEG) to study functional brain networks in neurodegenerative disorders. J Neurol Sci 2010;289:128-34

101. Tormenti M, Krieger D, Puccio AM, et al. Magnetoencephalographic virtual recording: a novel diagnostic tool for concussion. Neurosurg Focus 2012;33:E9

102. Huang M-X, Nichols S, Robb A, et al. An automatic MEG lowfrequency source imaging approach for detecting injuries in mild and moderate TBI patients with blast and non-blast causes. Neuroimage 2012;61:1067-82

103. Huang MX, Theilmann RJ, Robb A, et al. Integrated imaging approach with MEG and DTI to detect mild traumatic brain injury in military and civilian patients. J Neurotrauma 2009;26:1213-26

104. Tarapore PE, Findlay AM, Lahue SC, et al. Resting state magneto- 
encephalography functional connectivity in traumatic brain injury. J Neurosurg 2013;118:1306-16

105. Verweij BH, Muizelaar JP, Vinas FC. Hyperacute measurement of intracranial pressure, cerebral perfusion pressure, jugular venous oxygen saturation, and laser Doppler flowmetry, before and during removal of traumatic acute subdural hematoma. J Neurosurg 2001;95:569-72

106. Adelson PD, Clyde B, Kochanek PM, et al. Cerebrovascular response in infants and young children following severe traumatic brain injury: a preliminary report. Pediatr Neurosurg 1997;26: $200-07$

107. Nedd K, Sfakianakis G, Ganz W, et al. 99mTc-HMPAO SPECT of the brain in mild to moderate traumatic brain injury patients: compared with CT: a prospective study. Brain Inj 1993;7:469-79

108. Kinuya K, Kakuda K, Nobata K, et al. Role of brain perfusion singlephoton emission tomography in traumatic head injury. Nucl Med Commun 2004;25:333-37

109. Gowda NK, Agrawal D, Bal C, et al. Technetium Tc-99m ethyl cysteinate dimer brain single-photon emission CT in mild traumatic brain injury: a prospective study. AJNR Am J Neuroradiol 2006;27:447-51

110. Pavel D, Jobe T, Devore-Best S, et al. Viewing the functional consequences of traumatic brain injury by using brain SPECT. Brain Cogn 2006;60:211-13

111. Shin YB, Kim SJ, Kim IJ, et al. Voxel-based statistical analysis of cerebral blood flow using Tc-99m ECD brain SPECT in patients with traumatic brain injury: group and individual analyses. Brain Inj 2006;20:661-67

112. Uruma G, Hashimoto K, Abo M. A new method for evaluation of mild traumatic brain injury with neuropsychological impairment using statistical imaging analysis for Tc-ECD SPECT. Ann Nucl Med 2013;27:187-202

113. Bavetta S, Nimmon CC, White J, et al. A prospective study comparing SPET with MRI and CT as prognostic indicators following severe closed head injury. Nucl Med Commun 1994;15:961-68

114. Abu-Judeh HH, Parker R, Singh M, et al. SPET brain perfusion imaging in mild traumatic brain injury without loss of consciousness and normal computed tomography. Nucl Med Commun 1999;20:505-10

115. Yamaki T, Imahori $Y$, Ohmori $Y$, et al. Cerebral hemodynamics and metabolism of severe diffuse brain injury measured by PET. J Nucl Med 1996;37:1166-70

116. Wintermark M, van Melle G, Schnyder P, et al. Admission perfusion CT: prognostic value in patients with severe head trauma. Radiology 2004;232:211-20

117. Wintermark M, Chiolero R, van Melle G, et al. Relationship between brain perfusion computed tomography variables and cerebral perfusion pressure in severe head trauma patients. Crit Care Med 2004;32:1579-87

118. Wintermark M, Chiolero R, Van Melle G, et al. Cerebral vascular autoregulation assessed by perfusion-CT in severe head trauma patients. J Neuroradiol 2006;33:27-37

119. Metting Z, Rodiger LA, de Jong BM, et al. Acute cerebral perfusion CT abnormalities associated with posttraumatic amnesia in mild head injury. J Neurotrauma 2010;27:2183-89

120. Soustiel JF, Mahamid E, Goldsher D, et al. Perfusion-CT for early assessment of traumatic cerebral contusions. Neuroradiology 2008;50:189-96

121. Garnett MR, Blamire AM, Corkill RG, et al. Abnormal cerebral blood volume in regions of contused and normal appearing brain following traumatic brain injury using perfusion magnetic resonance imaging. J Neurotrauma 2001;18:585-93

122. Liu W, Wang B, Wolfowitz R, et al. Perfusion deficits in patients with mild traumatic brain injury characterized by dynamic susceptibility contrast MRI. NMR Biomed 2013;26:651-63

123. Ge YL, Patel MB, Chen Q, et al. Assessment of thalamic perfusion in patients with mild traumatic brain injury by true FISP arterial spin labelling MR imaging at 3T. Brain Inj 2009;23:666-74
124. Kim J, Whyte J, Patel S, et al. Resting cerebral blood flow alterations in chronic traumatic brain injury: an arterial spin labeling perfusion FMRI study. J Neurotrauma 2010;27:1399-411

125. Peters AM, Gunasekera RD, Henderson BL, et al. Noninvasive measurement of blood flow and extraction fraction. $\mathrm{Nucl} \mathrm{Med} \mathrm{Com-}$ mun 1987;8:823-37

126. Wintermark M, Thiran JP, Maeder P, et al. Simultaneous measurement of regional cerebral blood flow by perfusion $\mathrm{CT}$ and stable xenon CT: a validation study. AJNR Am J Neuroradiol 2001;22: 905-14

127. Kudo K, Terae S, Katoh C, et al. Quantitative cerebral blood flow measurement with dynamic perfusion $\mathrm{CT}$ using the vascular-pixel elimination method: comparison with $(\mathrm{H} 2 \mathrm{O})-\mathrm{O}-15$ positron emission tomography. AJNR Am J Neuroradiol 2003;24:419-26

128. Latchaw RE, Yonas H, Pentheny SL, et al. Adverse reactions to xenon-enhanced CT cerebral blood flow determination. Radiology 1987;163:251-54

129. Wintermark M, Reichhart M, Thiran JP, et al. Prognostic accuracy of cerebral blood flow measurement by perfusion computed tomography, at the time of emergency room admission, in acute stroke patients. Ann Neurol 2002;51:417-32

130. Wintermark M, Reichhart M, Cuisenaire O, et al. Comparison of admission perfusion computed tomography and qualitative diffusion- and perfusion-weighted magnetic resonance imaging in acute stroke patients. Stroke 2002;33:2025-31

131. Amorim RL, Bor-Seng-Shu E, Gattás GS, et al. Decompressive craniectomy and cerebral blood flow regulation in head injured patients: a case studied by perfusion CT. J Neuroradiol 2012;39:346-49

132. Metting Z, Cerliani L, Rodiger LA, et al. Pathophysiological concepts in mild traumatic brain injury: diffusion tensor imaging related to acute perfusion CT imaging. PLoS One 2013;8:e64461

133. Grossman EJ, Jensen JH, Babb JS, et al. Cognitive impairment in mild traumatic brain injury: a longitudinal diffusional kurtosis and perfusion imaging study. AJNR Am J Neuroradiol 2013;34:95157, S1-3

134. Stamatakis EA, Wilson JTL, Hadley DM, et al. SPECT imaging in head injury interpreted with statistical parametric mapping. J Nucl Med 2002;43:476-83

135. Lorberboym M, Lampl Y, Gerzon I, et al. Brain SPECT evaluation of amnestic ED patients after mild head trauma. Am J Emerg Med 2002;20:310-13

136. Lewine JD, Davis JT, Bigler ED, et al. Objective documentation of traumatic brain injury subsequent to mild head trauma: multimodal brain imaging with MEG, SPECT, and MRI. $J$ Head Trauma Rehabil 2007;22:141-55

137. Chen SHA, Kareken DA, Fastenau PS, et al. A study of persistent post-concussion symptoms in mild head trauma using positron emission tomography. J Neurol Neurosurg Psychiatry 2003;74:326-32

138. Peskind ER, Petrie EC, Cross DJ, et al. Cerebrocerebellar hypometabolism associated with repetitive blast exposure mild traumatic brain injury in 12 Iraq war veterans with persistent post-concussive symptoms. Neuroimage 2011;54(suppl 1):S76-82

139. Byrnes KR, Wilson CM, Brabazon F, et al. FDG-PET imaging in mild traumatic brain injury: a critical review. Front Neuroenergetics 2014;5:13

140. Raji CA, Tarzwell R, Pavel D, et al. Clinical utility of SPECT neuroimaging in the diagnosis and treatment of traumatic brain injury: a systematic review. PLoS One 2014;9:e91088

141. Bigler ED. Neuroimaging biomarkers in mild traumatic brain injury (mTBI). Neuropsychol Rev 2013;23:169-209

142. Hong YT, Veenith T, Dewar D, et al. Amyloid imaging with carbon 11-labeled Pittsburgh compound B for traumatic brain injury. JAMA Neurol 2014;71:23-31

143. Small GW, Kepe V, Siddarth P, et al. PET scanning of brain tau in retired National Football League players: preliminary findings. Am J Geriatr Psychiatry 2013;21:138-44 
144. Stein TD, Alvarez VE, McKee AC. Chronic traumatic encephalopathy: a spectrum of neuropathological changes following repetitive brain trauma in athletes and military personnel. Alzheimers Res Ther 2014;6:4

145. Gavett BE, Stern RA, McKee AC. Chronic traumatic encephalopathy: a potential late effect of sport-related concussive and subconcussive head trauma. Clin Sports Med 2011;30:179-88, xi

146. Shively S, Scher AI, Perl DP, et al. Dementia resulting from traumatic brain injury: what is the pathology? Arch Neurol 2012; 69:1245-51

147. McKinney A, Ott F, Short J, et al. Angiographic frequency of blunt cerebrovascular injury in patients with carotid canal or vertebral foramen fractures on multidetector CT. Eur J Radiol 2007;62: 385-93

148. Franz RW, Willette PA, Wood MJ, et al. A systematic review and meta-analysis of diagnostic screening criteria for blunt cerebrovascular injuries. J Am Coll Surg 2012;214:313-27

149. Biffl WL, Moore EE, Offner PJ, et al. Blunt carotid arterial injuries: implications of a new grading scale. J Trauma 1999;47:845-53

150. Löhrer L, Vieth V, Nassenstein I, et al. Blunt cerebrovascular injuries in acute trauma care: a screening protocol. Eur Spine J 2012;21:837-43

151. Cothren CC, Moore EE, Ray CE Jr, et al. Cervical spine fracture patterns mandating screening to rule out blunt cerebrovascular injury. Surgery 2007;141:76-82

152. Kopelman TR, Leeds S, Berardoni NE, et al. Incidence of blunt cerebrovascular injury in low-risk cervical spine fractures. Am J Surg 2011;202:684-88; discussion 688-89

153. Burlew CC, Biffl WL, Moore EE, et al. Blunt cerebrovascular injuries: redefining screening criteria in the era of noninvasive diagnosis. J Trauma Acute Care Surg 2012;72:330 -35; discussion 33637, quiz 539

154. Berne JD, Cook A, Rowe SA, et al. A multivariate logistic regression analysis of risk factors for blunt cerebrovascular injury. J Vasc Surg 2010;51:57-64

155. Chokshi FH, Munera F, Rivas LA, et al. 64-MDCT angiography of blunt vascular injuries of the neck. AJR Am J Roentgenol 2011;196:W309-15

156. Desai NK, Kang J, Chokshi FH. Screening CT angiography for pediatric blunt cerebrovascular injury with emphasis on the cervical “seatbelt sign.” AJNR Am J Neuroradiol 2014;35:1836-40

157. Dhillon RS, Barrios C, Lau C, et al. Seatbelt sign as an indication for four-vessel computed tomography angiogram of the neck to diagnose blunt carotid artery and other cervical vascular injuries. $\mathrm{Am}$ Surg 2013;79:1001-04

158. Fleck SK, Langner S, Baldauf J, et al. Blunt craniocervical artery injury in cervical spine lesions: the value of CT angiography. Acta Neurochir (Wien) 2010;152:1679-86

159. Liang T, McLaughlin PD, Louis L, et al. Review of multidetector computed tomography angiography as a screening modality in the assessment of blunt vascular neck injuries. Can Assoc Radiol J 2013;64:130-39

160. Patterson $\mathrm{BO}$, Holt $\mathrm{PJ}$, Cleanthis $\mathrm{M}$, et al. Imaging vascular trauma. Br J Surg 2012;99:494-505

161. Sliker CW. Blunt cerebrovascular injuries: imaging with multidetector CT angiography. Radiographics 2008;28:1689-708; discussion $1709-10$

162. Shiroff AM, Gale SC, Martin ND, et al. Penetrating neck trauma: a review of management strategies and discussion of the 'No Zone' approach. Am Surg 2013;79:23-29

163. Kansagra AP, Cooke DL, English JD, et al. Current trends in endovascular management of traumatic cerebrovascular injury. $J \mathrm{Neu}$ rointerv Surg 2014;6:47-50

164. Hassan AE, Zacharatos H, Souslian F, et al. Long-term clinical and angiographic outcomes in patients with cervico-cranial dissections treated with stent placement: a meta-analysis of case series. J Neurotrauma 2012;29:1342-53 